\title{
Recurrent renal cell cancer: 10 years or more after nephrectomy
}

\author{
Emmanuel Abara, MB, FRCSC, FACS, ${ }^{*}$ Iolanda Chivulescu; ${ }^{\dagger}$ Nilam Clerk, MD, FRCPC, ${ }^{\ddagger}$ Pablo Cano, MD, \\ FRCPC; ; Alexis Goth, MD, CCFP
}

\begin{abstract}
Localized renal cell carcinoma (RCC) responds well to surgery. Patients often question how long they have to be on surveillance after their surgery. Several follow-up patterns have been described in the literature. Until 2009, no published established Canadian guidelines existed to assist Canadian health-care practitioners in the surveillance of these patients. We present 3 cases of RCC that recurred 10 years or longer after the initial nephrectomy. These cases emphasize the need for careful long-term follow-up, as recommended in the Canadian Urological Association guidelines. We also discuss the optimism of prolonged disease survival in the era of novel therapeutic agents that target angiogenesis.
\end{abstract}

Can Urol Assoc J 2010;4(2):E45-E49

\section{Introduction}

The widespread use of relatively non-invasive diagnostic imaging studies has resulted in early detection of renal cell carcinoma (RCC) in asymptomatic individuals. Most patients have low-volume, low-stage sporadic clear cell RCC that responds well to surgery. ${ }^{1-6}$ Despite this, at the time of initial diagnosis a third of patients already have metastatic disease; of the remaining two-thirds of patients, about $30 \%$ to $40 \%$ of them will have distant metastases or recurrences during follow-up. ${ }^{2-6}$

With the introduction of therapies that target tumour angiogenesis, the outlook of metastatic and recurrent RCC is changing. With this change comes the need for good surveillance and early detection. A few published guidelines on follow-up exist in the English literature, some of which suggest individualized protocols and cost-containment. ${ }^{1-5}$ The recently published Canadian Urological Associaton (CUA) guideline is a welcome addition. ${ }^{7}$

Our patients were seen every 3 months for the first year, then every 6 months for the second and third years and annually thereafter. Assessment included physical examinations, blood pressure measurements, serum creatinine, urinalysis and abdominal ultrasound. Chest x-rays were done annually. Computed tomography (CT) scans were ordered every 6 months for a high-grade tumour or as required based on ultrasound findings.

We present 3 cases of recurrent RCC, 10 years or longer after the initial nephrectomy. These cases emphasize the need for adequate surveillance and care in the era of new therapies for metastatic and/or recurrent RCC.

\section{Case 1}

In 1993, a 46-year-old woman, para 1, was referred with an ultrasound diagnosis of a $5 \mathrm{~cm}$ right renal mass, discovered during the course of a gastrointestinal disease investigation. She had no urinary symptoms. There was a history of hiatus-hernia, cesarean section and hysterectomy.

Physical examination was normal, and no abdominal masses were palpable. Blood pressure was 110/70 $\mathrm{mmHg}$. Urinalysis revealed, 3 to 5 white blood cells (WBC) per high power field (HPF), 11 to 20 epithelial cells/HPF and no erythrocytes. Hemoglobin was $143 \mathrm{~g} / \mathrm{L}$, serum creatinine was $82 \mathrm{mmol} / \mathrm{L}$ and electrolytes and liver function tests were normal. Intravenous pyelogram (IVP), abdominal CT scan and renal angiogram suggested this mass as neoplastic. She had a successful right thoraco-abdominal nephrectomy. The 5-cm mass was cortical, partly exophytic distorting the renal collecting system. The capsular, renal, vascular, ureteric margins, perinephric tissues, regional lymph nodes and adrenal glands were free of tumour. Microscopy showed cystic clear cell RCC, Fuhrman nuclear grade I (Fig. 1a).

Active surveillance was instituted. Between 1997 and 2005 there were no urological events reported, until she developed a symptomatic ureteric stone in July 2005. Left ureterscopic lithotripsy was performed by her local urologist. She was stone-free, but had persistent microhematuria.

She was referred to one of the authors (EA) for a second opinion in 2006 - 13 years after the right nephrectomy. Abdominal ultrasound and CT scan showed a $5-\mathrm{cm}$ solid mass in the lower pole of the solitary left kidney. Chest $x-$ ray was normal. There was no palpable mass on examina- 


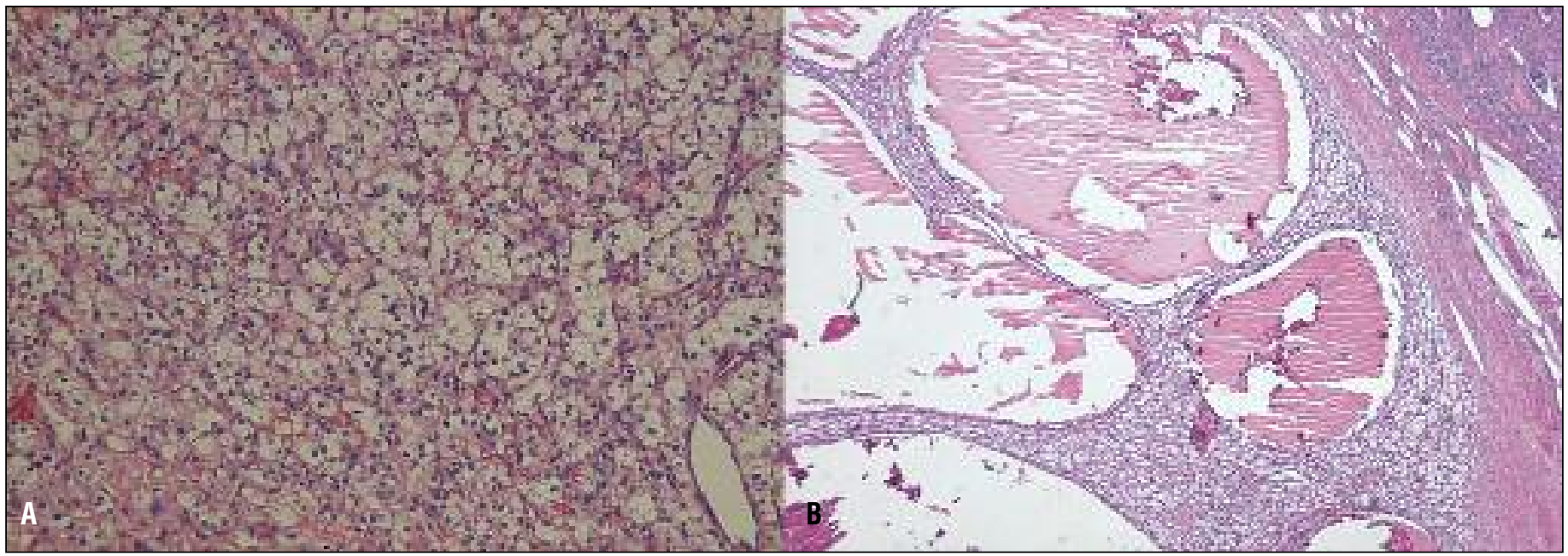

Fig. 1a. For case 1, in 1993, solid Fuhrman grade I (image A), cystic area (image B).

tion. Liver and renal function studies were normal with a serum creatinine of $74 \mathrm{mmol} / \mathrm{L}$. Hemoglobin was $128 \mathrm{~g} / \mathrm{L}$.

A review of the case with a second radiologist and urologist was concurrent with a solid $5 \times 4 \mathrm{~cm}$ solid mass in the lower pole of the left solitary kidney. There were no lesions in the liver, lungs and lymph nodes.

An open and/or laparoscopic partial nephrectomy and institutions of care were discussed. She was advised that dialysis may be necessary if she became anephric. Following informed consent, she had open left partial nephrectomy. She made satisfactory recovery and was discharged 4 days after the operation. The pathology report described the tumour as localized cystic clear cell RCC; $5.5 \mathrm{~cm}$ in greatest diameter with Fuhrman nuclear grade I (Fig. 1b).

Following discharge from the hospital, the patient was referred to a medical oncologist and a nephrologist. On surveillance, there has been no evidence of disease 4 years following left partial nephrectomy and 17 years post right nephrectomy for cystic clear cell RCC.

\section{Case 2}

In 1995, a 50-year-old man was referred for gross painless hematuria, weakness, lower back pain, $30 \mathrm{lb}$ weight loss in a year and erectile dysfunction. He did not smoke, but drank alcohol occasionally. He had some lower urinary tract symptoms (LUTS). On examination, he was not pale. $\mathrm{He}$ had a large palpable right-sided abdominal mass. On digital rectal examination, the prostate felt benign and enlarged to about $30 \mathrm{cc}$. He had no neurological or musculoskeletal defects. An abdominal ultrasound and IVP revealed a $10-\mathrm{cm}$ solid mass obliterating the right psoas muscle and splaying the calyces in the lower pole of the kidney. Multiple bladder diverticulae and poor bladder emptying were demonstrated. Hemoglobin was $154 \mathrm{~g} / \mathrm{L}$. Liver function tests, WBC, platelets and serum calcium were normal. A CT scan of the abdomen and pelvis confirmed a large $10-\mathrm{cm}$ renal mass involving the middle and lower poles. The tumour appeared to be involving Gerota's fascia. There

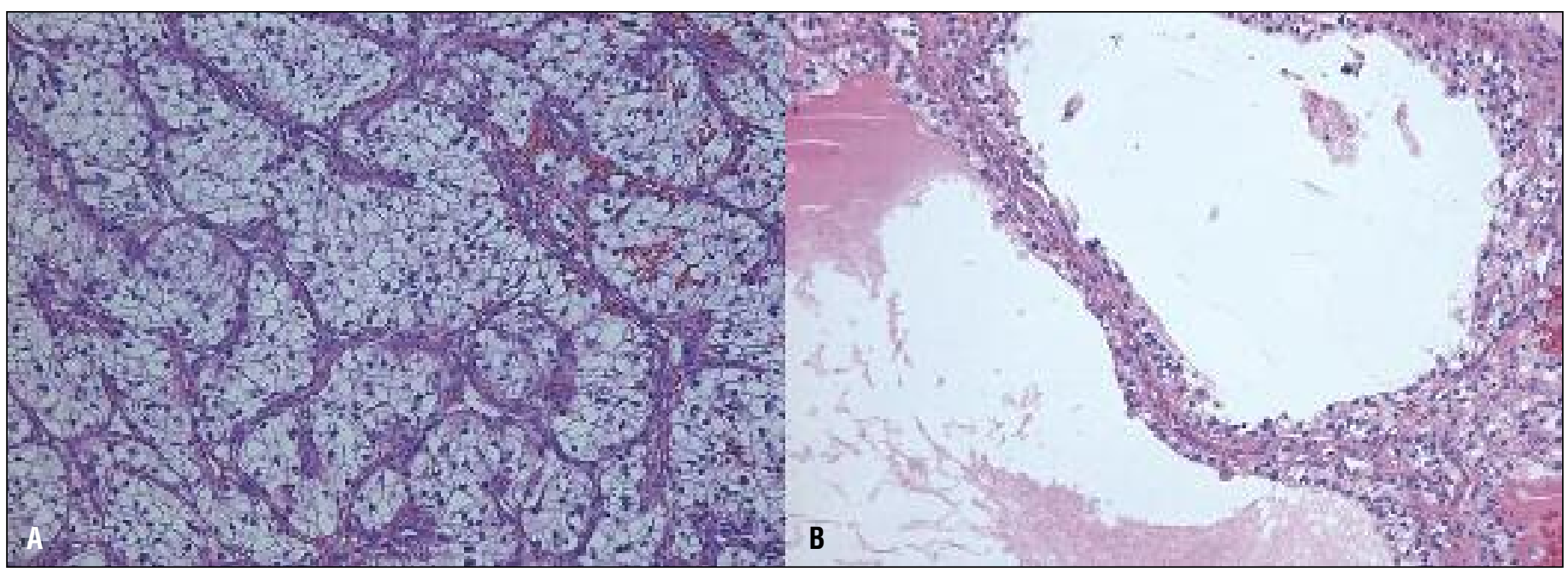

Fig. $\mathbf{1 b}$. For case 1, in 2006, clear cell renal cell carcinoma, 10x magnification (image A), area with cystic architecture (image B). 


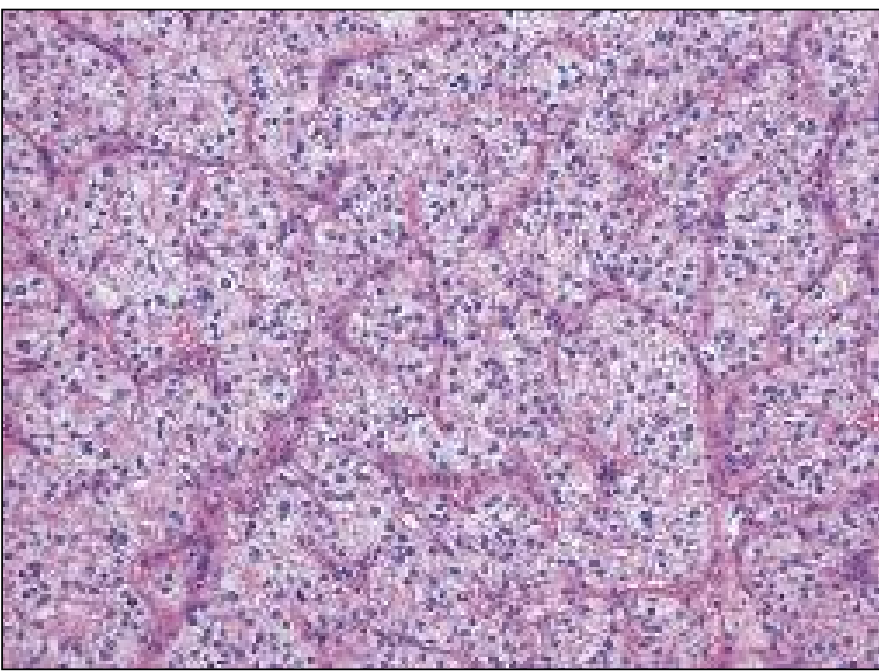

Fig. 2a. For case 2, clear cell renal cell carcinoma, Fuhrman grade I at 10x magnification.

were no retroperitoneal lymphadenopathy and no other organ involvement. Renal angiogram reported 2 renal arteries, neovascularity in the mid and lower poles. Renal arteriogram did not opacify the renal vein. Inferior venacavogram showed no tumour thrombus in the cava. Informed consent was obtained for radical nephrectomy. At the time of the exploration, there was tumour thrombus in the renal vein. Right thoraco-abdominal nephrectomy with excision of cuff of inferior venacava and paracaval lymphadenectomy was done. Postoperativly, he did very well and was discharged 4 days after the operation. At this time, we began active surveillance.

Grossly, the specimen, $17 \times 7 \times 8$, weighed $700 \mathrm{~g}$; bivalve, it showed a cavitated yellowish to tan-coloured mass arising in the lower and mid pole extending into the surrounding adipose tissue. An intact capsule separated the mass from the adipose tissue. There was a tumour thrombus in the renal vein about $1.5 \mathrm{~cm}$ away from the resection margin.

Microscopically, the tumour was mostly well-differentiated clear cell RCC with areas of infarction, necrosis, fibrosis and pseudo-capsular formation. The resection margins were free of tumour. The remaining parenchyma showed normal architecture with no arteriolonephrosclerosis (Fig. 2).

The patient was assessed as scheduled until November 1998, when his family physician relocated. For several years after this time he had no family physician. He felt well and did not keep his urology appointments. In 2006, he developed bothersome LUTS and found a new family doctor who investigated this development. Urine culture grew Staphylococcus saprophyticus. His serum prostate-specific antigen (PSA) was $20 \mathrm{ng} / \mathrm{mL}$ and his creatinine was $135 \mu \mathrm{mol} / \mathrm{L}$. Abdominal and pelvic ultrasound showed urinary retention and masses in the right renal bed, solitary left kidney and pancreatic bed. These were confirmed by CT scans (Fig. 3).

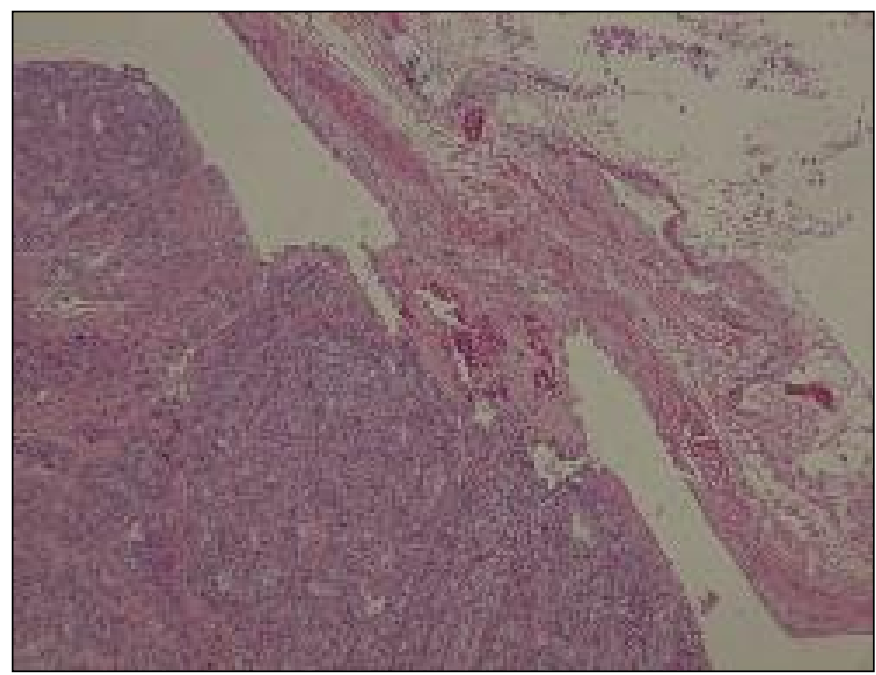

Fig. 2 b. For case 2, clear cell renal cell carcinoma with renal vein involvement.

He was referred to a medical oncologist at the regional cancer centre. After trying several agents, the patient responded well to sorafenib tosylate $200 \mathrm{mg}, 3$ times a day. Transurethral resection of the prostate relieved his obstruction. He was free of infection with a normal serum creatinine and PSA. On sorafenib tosylate $200 \mathrm{mg}$, 2 times a day, there has been no disease progression, 4 years after the discovery of the recurrent tumour and 15 years post right nephrectomy.

\section{Case 3}

In 1984, a 40-year-old man presented with right flank pain and hematuria. Intravenous pyelogram and abdominal ultrasound suggested a right renal mass. Renal angiogram confirmed a vascular tumour localized within the kidney. Right thoraco-abdominal nephrectomy was done and patient was placed on a surveillance program.

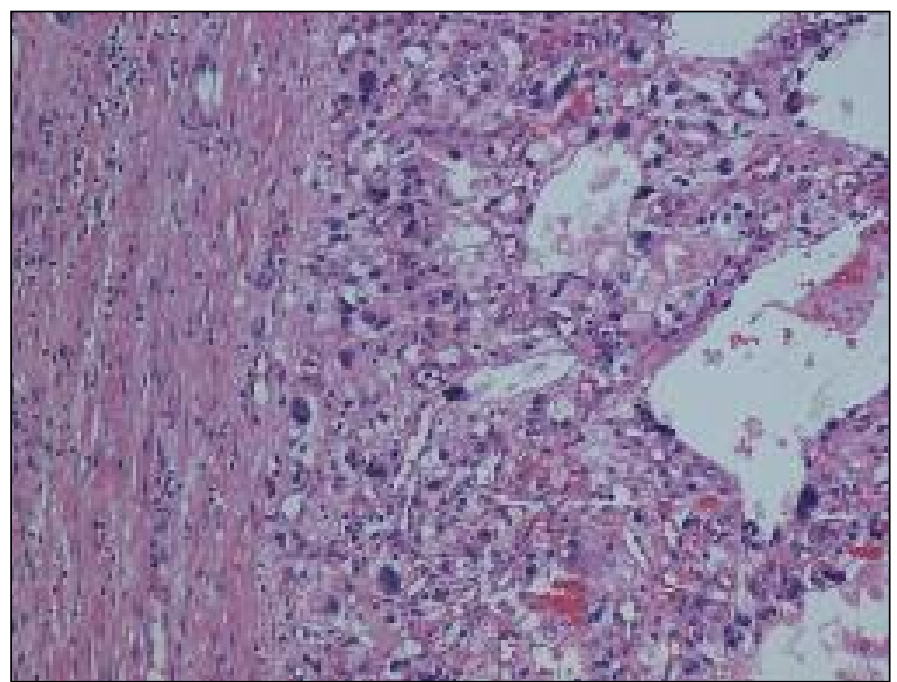

Fig 2c. For case 2, areas with high-grade features, Fuhrman grade III-IV. 


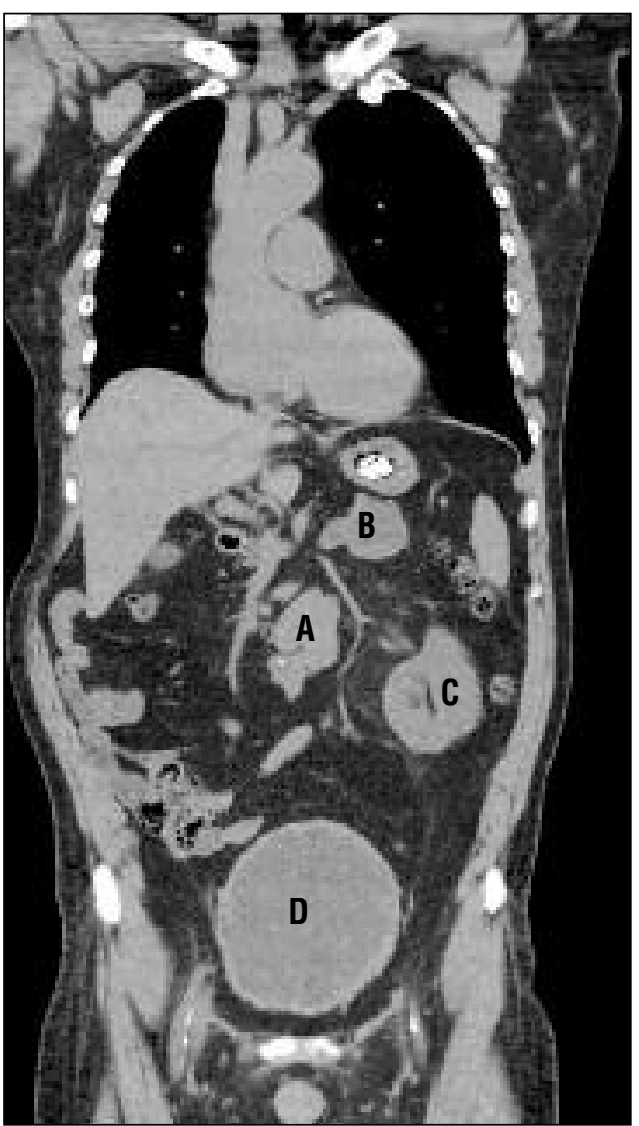

Fig. 3. A: Tumour in right renal fossa; $B$ : tumour in the pancreatic bed; C: tumour in the solitary left kidney D: urinary bladder retention.

In 1989, his urologist retired and transferred care to one of the authors (EA) who continued yearly surveillance with chest x-ray, abdominal and pelvic ultrasound, urinalysis and clinical examination.

In 1995, the chest x-ray showed a mass in the right hemithorax; a CT scan of the chest, abdomen, pelvis and CT-guided biopsy confirmed metastatic RCC in the right pleura and a normal solitary left kidney. The retroperitoneal and intraperitoneal organs were free of metastatic disease. The patient was referred to a thoracic surgeon at the regional cancer centre and had a thoracotomy and an excision of the tumour. He was treated with cytokine therapy (interferon- $\alpha$ ). Adequate follow-up was not available.

\section{Discussion}

Following surgery for RCC, our patients often question how they are going to cope with one kidney. They worry about possible recurrence in the other kidney, how often they need to be assessed and for how long. It is generally agreed that these patients should be followed in the postoperative period not just for possible recurrent or metastatic disease, but for other reasons, such as renal function and blood pressure. Pre-existing or concomitant parenchymal kidney disease requires stricter follow-up. Partial nephrectomy in a solitary kidney raises the risk for potential progressive renal impairment due to glomerular sclerosis as a result of hyperfiltration in a reduced renal mass. ${ }^{1}$ The patient from case 1 is currently under this type of surveillance.

Several authors have emphasized the need to individualize surveillance based on tumour stage, grade, tumour volume and type of surgery, whether partial or total nephrectomy. ${ }^{1-5}$ Ljungberg and colleagues state that tumour stage is the best predictor of prognosis; these authors used it with tumour grade and DNA ploidy to propose an individualized cost-conscious follow-up protocol. ${ }^{3}$

Time to disease recurrence or progression is important in planning surveillance. In the literature, most of the tumour recurrences occur within 5 years. ${ }^{1-5}$ Some authors advocate follow-up for the first 5 years, others for 10 years. Given the various case reports of recurrence 10 years or longer after the initial nephrectomy ${ }^{8-11}$ and our 3 cases, it would appear that follow-up for life is reasonable. However, this surveillance pattern should be individualized and cost-conscious.

As several authors have pointed out, RCC has a notoriety of spreading to unusual sites. ${ }^{1-4,8,10-12}$ However, the common sites, such as lungs, liver, brain and bone, can be monitored easily and there are reports of successful resection of isolated metastases from these organs.

Contralateral kidney involvement with tumour, as in the patient in case 1 , has been reported to occur in $0.4 \%$ to $12.9 \%{ }^{13}$ Positive surgical margins and multifocality were good predictors in clear cell RCC and nuclear grade for recurrent papillary RCC. ${ }^{13}$ The patient in case 1 had a 5 -cm tumour, of cystic clear cell variety, with margins negative and no multifocality relapse in the solitary left kidney 13 years after the initial right nephrectomy. She is currently disease-free 4 years after the partial nephrectomy. The rarity of this case is supported in the literature. $1,8,13$ This case underscores the need for judicious follow-up of the abdomen. We have found ultrasound easily accessible and relatively non-invasive in early detection even in remote area hospitals. When detected early, small tumours are better defined with a CT scan and are amendable to partial nephrectomy. Preservation of renal function and elimination of cancer are achievable.

Computed tomography scans are the preferred diagnostic imaging modality for patients with retroperitoneal relapse, tumours larger than $10 \mathrm{~cm}$, involvement of perinephric fat, renal vein (as in the patient in case 2 ) and regional lymph nodes. ${ }^{1-4}$

The patient in case 3, followed with yearly chest x-rays, was found to have pulmonary metastasis 11 years postnephrectomy. A CT scan defined the lesion, thoracotomy and tumour excision followed by interferon- $\alpha$ therapy resulted in some survival benefit. This benefit would appear to justify the use of chest x-ray in the surveillance of these patients.

Occasional long-term survivors after the resection of isolated or multiple pulmonary lesions in combination with 
interleukin 2-based immunotherapy have been reported. ${ }^{1,11}$

The introduction of target therapy against the vascular endothelial growth factor pathway and related elements has opened a new horizon in the management of metastatic renal cancer. Agents, such as sunitinib, bevacizumab plus interferon, sorafenib and tensirolimus, have been used in several clinical settings in the management of metastatic or recurrent RCC. ${ }^{14-18}$

The patient from case 2 had disease in the right renal bed, solitary left kidney and pancreatic bed, 11 years postnephrectomy; he is alive 4 years with no disease progression on sorafenib therapy, after attempts with sunitinib and tensirolimus produced side-effects and logistic problems. He now uses sorafenib 200 mg orally twice daily and is being followed by his medical oncologist, urologist and family physician. While the debate goes on regarding who administers systemic non-cytotoxic kidney cancer therapies, ${ }^{17}$ the patient has disease progression-free interval with this collaborative care team approach. This approach is desirable and beneficial.

\section{Conclusion}

Based on our cases and the literature, long-term follow-up is necessary for early detection and management of recurrent RCC. When possible, nephron-sparing procedures provide survival advantage with the preservation of renal function. Surveillance programs should follow a protocol, such as the CUA guidelines, as per their jurisdiction of medical practice. ${ }^{6,7}$ This program should be individualized, tumourstage specific and cost-conscious. The advent of new oral agents that target tumour angiogenesis may not be curative, but these agents can lead to prolonged life.

${ }^{*}$ Richmond Hill Urology Practice \& Prostate Institute, Richmond Hill, ON; ${ }^{\dagger}$ McMaster University, Faculty of Science, Hamilton, ON; $¥$ Y ork Central Hospital, Richmond Hill, ON; §Northeastern Ontario Regional Cancer Centre, Sudbury, ON; *Sensenbrener Hospital, Kapuskasing, ON

\section{References}

1. Montie JE. Follow-up after partial and total nephrectomy for renal cell carcinoma. Urol Clin North Am 1994:21:589-92.

2. Hafez KS, Novick AC, Campbell SC. Patterns of tumour recurrence and guidelines for follow-up after nephron-sparing surgery for sporadic renal cell carcinoma. J Urol 1997;157:2067-70.

3. Liungberg B, Alamdari Fl, Rasmuson T, et al. Follow-up guidelines for non-metastatic renal cell carcinomo based on the occurrence of metastases after radical nephrectomy. BJU Int 1999;84:405-11.

4. Stephenson AJ, Chetner MP, Rourke K, et al. Guidelines for the surveillance of localized renal cell carcinoma based on the patterns of relapse after nephrectomy. J Urol 2004;172:58-62.

5. Liungberg B, Hanbury DC, Kuczyk MA, et al. Renal cell carcinoma guideline. Eur Uro 2007;51:1502-10.

6. Wood LA, Jewett MAS, eds. Renal cell carcinoma 2007 update. Can Urol Assoc J 2007; 1 (suppl): 51-76.

7. Kassouf W, Siemens R, Morash C, et al. Follow-up guidelines after radical or partial nephrectomy for localized and locally advanced renal cell carcinoma. Can Urol Assoc J 2009;3:73-6.

8. Roser F, Rosahl SK, Samil M. Single cerebral metastasis 3 and 19 years after primary renal cell carcinoma: case report and review of the literature. J Neurol Neurosurg Psychiatry 2002;72:257-8.

9. Riviello $C$, Tanini I, Cipriani $G$, et al. Unusual gastric and pancreatic metastasis renal cell carcinoma presentation 10 years after surgery and immunotherapy: a case report and a review literature. World J Gastroenterol 2006;12:5234-6.

10. Lordan JT, Fawcett WJ, Karanija ND. Solitary liver metastasis of chromophobe renal cell carcinoma 20 years after nephrectomy treated by hepatic resection. Urology 2008;72:230.

11. Nagai $T$, Igase $M, O c h i ~ M$, et al. Multiple metastases from renal carcinoma 15 years after nephrectomy. Nippon Ronene Iqakkai Zasshi 2007;44:747-51.

12. Shiraishi $\mathrm{K}$, Mohri J, Inoue $\mathrm{R}$, et al. Metastatic renal cell carcinoma to the bladder 12 years after radical nephrectomy. Int I Urol 2004;10:453-5.

13. Bani-Hani AH, Leibovich $B C$, Lohse $C M$, et al: Association with contralateral recurrence following nephrectomy for renal cell carcinoma using a cohort of 2352 patients. J Urol 2005; 173:391-4.

14. Levy DA, Swanson DA, Slaton JW, et al. Timely delivery of biological therapy after cytoreductive nephrectomy in carefully selected patients with metastatic renal cell carcinoma. J Urol 1998;159:1168-73.

15. Riechelmann RP, Chin S, Wang L, et al. Sorafenib for metastatic renal cancer: The Princess Margaret experience. Am J Clin Oncol 2008;31:182-7.

16. Herrman E, Gerss J, Bierer S, et al. Pre-treatment global quality of health predicts progression free survival in metastatic kidney cancer patients treated with sorafenib or sunitinib; I Cancer Res Clin Oncol 2009; 135:61-7

17. Klotz L. Sunitinib, sorafenib and other systemic non cytotoxic kidney cancer therapies can and should be administered by urologists. Can Urol Assoc J 2007; 1 (suppl 2):569-70.

18. Bielogrlic SK, Radulovic S, Babovic N. Molecular targeting agents in renal cell carcinoma: present strategies and future perspectives. Curr Pharm Des 2008; 14:1058-77.

Correspondence: Dr. Emmanuel Abara, Richmond Hill Urology Practice \& Prostate Institute, 9755 Bathurst St., Richmond Hill, ON L4C 3X5; rhuppi@rogers.com

Competing interests: None declared.

This paper has been peer-reviewed. 